\title{
Employment Changes, the Structure of Adjustment Costs, and Plant Size ${ }^{\dagger}$
}

\author{
Øivind A. Nilsen ${ }^{*}$ \\ (University of Bergen, and IZA-Bonn)
}

Kjell G. Salvanes

(Norwegian School of Economics and Business Administration, and IZA-Bonn)

Fabio Schiantarelli

(Boston College, and IZA-Bonn)

November 2, 2003

\begin{abstract}
In this paper we analyze the pattern of employment adjustment using a rich panel of Norwegian plants. The data suggest that the frequency of episodes of zero net employment changes is inversely related to plant size. We develop and estimate a simple " $q$ " model of labor demand, allowing for the presence of fixed, linear and convex components of adjustment costs. The econometric evidence supports the existence of purely fixed components, unrelated to plant size. As a result, the range of inaction is wider for smaller plants. The quadratic components of costs are also important. Finally, in most specifications both fixed and convex costs are higher for employment contractions.
\end{abstract}

JEL classification: D21, C23, E24

Key words: Adjustment costs, employment demand, size.

$\dagger$ We would like to thank Francis Kramarz, Julia Lane, Kevin Lang, Arthur Lewbel and participants to the ESEM'01 and EALE'02 conferences, and to seminars participants at IZA-Bonn, Humboldt University, DIWBerlin, BIRC-Maastricht University, University of Mannheim, University of Bergen, Norwegian School of Economics and Business Administration, CREST-INSEE, "Firms' Dynamic Adjustment"-workshop in BergamoItaly, and CAED'01-Aarhus for useful comments and suggestions.

* Corresponding author: Address; Department of Economics, University of Bergen, Fosswinckelsgt. 6, NO-5007 Bergen, Norway. Phone; +47 55589228, Fax; +47 55589210, E-mail: oivind.nilsen@econ.uib.no. 


\section{Introduction}

In the last few years there has been a heightened awareness of the shortcomings of traditional models of factor demand based on convex and symmetric adjustment costs and of the need to consider more general adjustment cost functions (see Hamermesh and Pfann (1996) for a critical review). The increased availability of firm and plant level panel data made it easier to provide empirical evidence on these issues and has lead to a blossoming of empirical studies, particularly on investment. ${ }^{1}$ Recent contributions on labor demand are scarcer, although by no mean absent. ${ }^{2}$

In this paper we intend to advance our understanding of the structure of employment adjustment costs, using a rich data set on Norwegian plants. In order to do that, we specify a simple optimizing model of labor demand that allows for a general structure of adjustment costs. In the basic specification, such costs are a function of net employment changes and include fixed, linear and quadratic components. ${ }^{3}$ The model is in the spirit of Abel and Eberly (1994) and can be thought of as a $q$ model for employment. Like other models of this type, it generates a region in which labor demand does not respond to changes in fundamentals, because the gains from increasing or decreasing employment by one unit is not large enough to compensate the incurring of adjustment costs. By including a truly fixed component in adjustment costs, we allow for the possibility that the range of the value of fundamentals for

\footnotetext{
${ }^{1}$ Among the most recent papers that analyze the importance of non convexities and irreversibility in generating non smooth investment patterns see Doms and Dunne (1998), Goolsbee and Gross (1997), Barnett and Sakellaris (1998), Abel and Eberly (1999), Cooper, Haltiwanger and Power (1999), Nilsen and Schiantarelli (2003), Letterie and Pfann (2002)

${ }^{2}$ See, the seminal contributions by Hamermesh $(1989,1992,1995)$, and the more recent ones by Bentolila and Saint-Paul (1994), Rota (1995), Abowd, Corbel and Kramarz (1999), Abowd and Kramarz (2003), Campbell and Fisher (2000a,b), Goux, Maurin and Pauchet (2001).

${ }^{3}$ In this paper, for simplicity sake, we specify adjustment costs as a function of net employment changes, consistently with many papers in the literature. For specifications based on both net and gross employment flows, see Hamermesh (1995). See also Kramarz and Michaud (2002) and Abowd and Kramarz (2003) for a model based on gross flows. This is an issue that deserves further investigation.
} 
which there are no employment changes may be wider for smaller plants (firms). Moreover, the response to fundamentals may differ for net employment increases versus decreases, reflecting asymmetries in adjustment costs.

The structure of the paper is as follows. In Section 2 we start by describing in details the Norwegian institutions regulating the adjustment of the labor factor. In Section 3 we present some descriptive evidence on employment adjustment patterns for plants in the machinery and metal product sector over the period 1986-1995. Section 4 contains a theoretical model that is potentially consistent with the descriptive statistics and that will underpin our econometric estimation. In Section 5 we present econometric estimates of various versions of the model. Section 6 concludes the paper.

\section{Institutional Determinants of Adjustment Costs}

The costs of changing employment are of course affected by the institutional setting and legislation introduced to protect workers against unfair dismissal. Both the rules regarding individual and collective dismissals, and the flexibility of plants with respect to temporary hiring and the use of subcontractors, are important in explaining the costs of adjustment for plants. The different types of constraints regulating the hiring and firing of workers are not completely transparent, since, in addition to national laws, collective agreements between employer and workers organization also are very important in regulating the adjustment of the labor factor. These agreements may differ across industries and workers, depending upon age, tenure, etc.

Two main laws govern the labor relations in Norway: the law on employment ("Sysselsettingsloven") and the law on labor relations (“Arbeidsmiljøloven”). The law on employment mainly regulates changes in labor during a period of restructuring and mass lay- 
offs by the firm. The latter was enacted in 1982 and it includes standards for general working conditions, overtime regulations and legal regulation for employment protection. According to the law on labor relations, dismissals for individual reasons are limited to cases of disloyalty, persistent absenteeism etc., while dismissals for economic reasons are automatically unfair. In general it is possible but very difficult to replace an individual worker in a given job with another worker. Hence, in general, there is a strong degree of employment protection in Norway. The law on employment states that the general rule for laying off a worker for economic reasons is that it layoffs can occur only when the job is "redundant" and the worker cannot be retained in another capacity. This regulation covers all workers independent of how long ago he/she has been hired. Requirements for collective dismissals in Norway basically follow the common minimum rules for EU-countries. It is important to notice is that a firm can dismiss workers not only when it is making losses but also when it has poor performance. There is not actually any rule on the selection of workers to be dismissed. However, the legal practice narrows down which workers can be dismissed. Conversations with lawyers in the employees' organizations indicate that a lot if not most of the cases of dismissals are taken to court, which is costly for the firms.

When it comes to other costs of dismissal, according to the employment law, employment is terminable with one month's notice in Norway for workers with tenure less or equal to five years. This one-month notice is at the lower end of the spectrum compared to many countries. However, most workers have a three months' notice requirement for both parties of the contract. Although there is no generalized legal requirement of severance pay in Norway, agreements in the private sector require lump-sum payments to workers who have reached age 50-55. As an example, in the contract between LO (the largest blue collar workers organization) and NHO (the employers' association), a worker who is 50 and has been working for 10 consecutive years or 20 years in the firms is eligible for one to two months 
pay. Comparable agreements exist for the other unions. Some EU-countries actually have even stronger job protection rules, including also general compensation, a social plan for re-training or transfer to another plant within a firm for instance. Although not mandatory, some of these other requirements are also commonplace in Norway. For this set of dismissal restriction, Norway is ranked slightly below average among OECD countries. Note finally that while some costs of reducing the workforce (such as redundancy payments) are related to the size of the reduction, others (such as advance notice requirements, legal and other administrative costs, etc.) may have significant fixed components.

The work force flexibility of an economy can be enhanced by allowing fixed-term contracts in addition to standard contract, and the use of temporary work agencies. In many OECD countries there has been a strong trend in liberalizing the use of these two schemes. In Norway, the use of fixed term contracts is allowed only for limited situations, such as specific projects, seasonal work or the replacement of workers who are absent temporary. However, it may not necessarily be as restrictive as it appears since defining a specific project for a firm is partly open to discretion. Repeated temporary contracts are possible with some limitations, and there is no rule limiting the cumulated duration of successive contract. In general the use of temporary work agencies are prohibited, but wide exceptions exists for service sector occupations. Restrictions for the number of renewals exist also here, and two years is the maximum for cumulated contracts. Compared to other OECD countries, Norway is ranked a little bit above average for the strictness of the use of temporary employment (OECD, 1999).

Very few comparative studies of the overall degree of employment protection exist. A much-sited study by Emerson (1987), ranks Italy as having the strongest employment protection rules while the UK and partly Denmark are at the other end of the spectrum. Norway is ranked together with Sweden, France and partly Germany (when all regulations are taken together) as an intermediate country with a fairly high degree of protection. Obviously 
inter-country comparisons are difficult. The most recent comparison was made by OECD in 1999, where Norway is ranked as number 12 out of 19 OECD countries for the late 1980s, and as number 19 out of 26 OECD countries for the late 1990s in the degree of restrictiveness (OECD, 1999). Evidence on the flexibility of the Norwegian economy from job and worker flows data suggests that it is about average for OECD countries, although worker flows are a bit below average. ${ }^{4}$ The overall impression is that legislation, contracts, and common practice impose important additional costs in Norway when adjusting the labor force downward, and possibly upward as well.

\section{Data and Descriptive Evidence}

\subsection{The Data}

Our empirical work is based on yearly plant level information for the period 1986-1995 contained in Manufacturing Statistics including production, sales, employment, production costs, the age of the plant, and whether the plant belongs to a multi-plant or single plant firm (see Halvorsen, Jenssen and Foyn (1991)). Total sales are calculated by adding sales of produced goods and traded goods, income from repairs and contracted works, income from deliveries and leasing to other plants in same firm. We are forced to restrict our attention to plants with at least five employees, since plant or firm specific information is not available for plants below five employees. We have excluded all auxiliary units which do not take part directly in production, such as separate storage and office units. Plants in which the central or local governments own more than 50 percent of the equity have been also excluded from the sample, as well as observations that are reported as "copied from previous year". This actually means that a data entry is missing. The remaining data were trimmed to remove outliers.

\footnotetext{
${ }^{4}$ See Salvanes (1997).
} 
In this paper we focus on plants in the metal products and machinery industries (ISIC 38), excluding large-construction and shipbuilding industry. Concentrating on a single industry reduces the heterogeneity problem in estimation. Moreover, sector 38 is an important one, accounting for $35 \%$ of manufacturing employment in 1990, and it contains a large number of plants of different size. Finally, we included only series with at least six consecutive observations, since one of the estimation methods requires the availability of a reasonable minimum number of years of data for implementation. The final unbalanced panel contains production plants with a total of 8414 observations from 1047 plants.

\subsection{Descriptive Statistics}

What are the basic patterns of employment changes for our sample of Norwegian plants? We summarize the basic characteristics of the distribution of net employment changes in Table 1. Employment increases and decreases occur with similar frequency: approximately $40 \%$ of the observations represent positive employment changes while $42 \%$ correspond to employment decreases. Interestingly in almost $18 \%$ of cases we observe no employment changes. This may be suggestive of the fact that changing the number of jobs even by a very small amount may imply sizeable adjustment costs that deter plants from adjusting. This would be the case for instance, in the presence of fixed or linear components of adjustment costs. If the plant increases the work force, the more frequent changes occur in the interval $(0$, $+20 \%$ ]. This occurs $30 \%$ of the times (unconditionally) or $71 \%$ of the time, conditional on the plant expanding, and it represents a 52\% share of total employment increases. Similarly, if the plant contracts, decreases in the interval $[-20 \%, 0$ ) occur $32 \%$ of the times (unconditionally) and $79 \%$ of the times, conditionally on the plant contracting, and it represents a $56 \%$ share of total employment decreases. Although changes in excess of plus or minus $20 \%$ are less frequent, the account (obviously) for close to half of employment increases or decreases. 
The pattern of adjustment differs in at least one important way across plants of different sizes. The frequency of episodes characterized by no employment changes decreases markedly with size. The frequency of zero episodes is approximately $25 \%$ for plants of 25 workers or less, $10 \%$ for plants of $25-50$ workers, $5 \%$ for plants of $51-100$ workers and $3 \%$ for plants larger than 100 workers. There are several reasons why there is a connection between plant size and the frequency of no employment change episodes. One possible explanation may be the existence of a pure fixed component of adjustment costs associated with net job contraction, net job expansion, or both. Such fixed component is a bigger relative burden for smaller plants and may induce more infrequent adjustments for such plants. For the very smallest of plants there may also be an element of indivisibility that generates consequences that are observationally equivalent to those of fixed costs. Indivisibility becomes, however, a less plausible explanation for plants of more than 25 workers, some of which continue to display significant occurrences of zero employment change episodes

\section{A Simple “ $q$ " Model for Employment}

In this section we develop a model for employment demand that allows for a general structure of adjustment costs and that is, at least in principle, consistent with the main feature of the data described above. The model is similar in spirit to $q$ models of investment in the presence of fixed adjustment costs and irreversibilities (see Abel and Eberly (1994, 1999)). It is also related to the model in Hamermesh (1992) that also contains fixed, linear, and quadratic components of adjustment costs. The linear and quadratic components are a function of net employment changes. In this model the growth rate of employment is related to the shadow value of the marginal worker, denoted by $q . q$ is the present discounted value of the marginal product of labor (which depends upon the structure of adjustment costs), net of wage costs. 
Our empirical strategy is to use simple approximations to $q$ and to estimate various versions of the model.

More precisely, we assume that plant $i$ maximizes the present discounted value of cash flow, defined as:

$$
V_{i t}=E_{t} \sum_{j=0}^{\infty} \beta^{j}\left[F\left(A_{i t+j}, L_{i t+j}\right)-w_{i t+j} L_{i t+j}-G\left(\Delta L_{i t+j}, L_{i t+j-1}\right)\right]
$$

where $L_{t i}$ denotes employment, $F\left(A_{i t}, L_{i t}\right)$ the gross production function, $A_{i i}$ is a technology shock, $w_{i t}$ the wage rate per worker, and $G\left(\Delta L_{i t}, L_{i t-1}\right)$ adjustment costs, assumed to be a function of net employment changes. ${ }^{5}$ Adjustment costs contain fixed, linear and quadratic components. Fixed costs, in turn, contain two elements: $a_{0}$, that is truly fixed, and $a_{1} L_{t-1}$ that depends upon plant size. More specifically:

$$
\begin{aligned}
G\left(\Delta L_{i t}, L_{i t-1}\right)= & D_{i t}^{+}\left[a_{0}^{+}+a_{1}^{+} L_{i t-1}+b^{+} \Delta L_{i t}+\frac{c^{+}}{2}\left(\frac{\Delta L_{i t}}{L_{i t-1}}\right)^{2} L_{i t-1}\right] \\
& +D_{i t}^{-}\left[a_{0}^{-}+a_{1}^{-} L_{i t-1}-b^{-} \Delta L_{i t}+\frac{c^{-}}{2}\left(\frac{\Delta L_{i t}}{L_{i t-1}}\right)^{2} L_{i t-1}\right]
\end{aligned}
$$

$D_{i t}^{+}\left(D_{i t}^{-}\right)$is a dummy that equals one when the plant expands (contracts) and it is zero otherwise. Similarly to Abel and Eberly $(1994,1999)$, when plants increase employment, the proportional increase in employment satisfies:

\footnotetext{
${ }^{5}$ Although capital is not introduced explicitly in the problem for ease of notation, plants should be thought as using both capital and labor. However, if capital is either not costly to adjust, or if its adjustment costs are additively separable from those for labor (there are no interrelated adjustment costs), then one would still obtain the same FOC's for labor. In our model we also abstract from the choice of hours worked.
} 


$$
\frac{\Delta L_{i t}}{L_{i t-1}}=\frac{1}{c^{+}}\left[q_{i t}-b^{+}\right]
$$

For employment to expand, it must be true that the marginal profits generated by the expansion are positive. This requires $q_{i t}$ to satisfy: $^{6}$

$$
q_{i t} \geq \sqrt{2 c^{+} \cdot\left(\frac{a_{0}^{+}}{L_{i t-1}}+a_{1}^{+}\right)}+b^{+}
$$

Similarly contractions in employment obey:

$$
\frac{\Delta L_{i t}}{L_{i t-1}}=\frac{1}{c^{-}}\left[q_{i t}+b^{-}\right]
$$

\section{Contractions occur when:}

$$
q_{i t} \leq-\sqrt{2 c^{-} \cdot\left(\frac{a_{0}^{-}}{L_{i t-1}}+a_{1}^{-}\right)}-b^{-}
$$

In all cases, the shadow value of employment, $q_{i t}$, is:

\footnotetext{
${ }^{6}$ The upper threshold value of $q_{i t}$ can be derived by finding the value of $q_{i t}$ at which an addition of $\Delta L_{i t}$ workers generates non-negative profits, i.e. $q_{i t} \cdot \Delta L_{i t} \geq a_{0}^{+}+a_{1}^{+} L_{i t-1}+b^{+} \Delta L_{i t}+\frac{c^{+}}{2}\left(\frac{\Delta L_{i t}}{L_{i t-1}}\right)^{2} L_{i t-1}$. Equation (3) is used to determine the optimal value of value of $\Delta L_{i t}$. The lower threshold for $q_{i t}$, when contracting the number of workers, is found in a similar fashion.
} 


$$
q_{i t}=E_{t} \sum_{j=0}^{\infty} \beta^{j}\left[F^{\prime}\left(A_{i t+j}, L_{i t+j}\right)-w_{i t+j}-\beta \cdot G_{L}\left(\Delta L_{i t+j}, L_{i t+j-1}\right)\right]
$$

$q_{i t}$ represents therefore the present discounted value of the marginal product of capital, net of adjustment costs, minus the flow of wage costs associated to the marginal worker.

In order to make the model estimable, we need to approximate the shadow value of a worker. We will assume that the latter is a linear function of the sales to labor ratio and of the wage. In the absence of an effect of the past stock of workers on adjustment costs, the marginal revenue product of labor would indeed be proportional to the sales to labor ratio if the production function is Cobb Douglas in labor (and capital). ${ }^{7}$ Moreover we assume that plants use simple $\mathrm{AR}(2)$ processes to forecast the sales to labor ratio and the wage rate. The main shortcut contained in this approximation is that we are abstracting from the fact that in partial derivative of the adjustment cost function with respect to the stock of workers, denoted by $G_{L}$, given net hiring (firing) is not zero in our model. In the context of models based only on quadratic adjustment costs, this has been a standard approximation in empirical work. ${ }^{8}$ When adjustment costs contain also a fixed component proportional to the stock of labor, one also abstracts from the effect of an extra worker on the expected present discounted value of these fixed costs. This is equal to the expected discounted sum of $a_{1}^{+}$and $a_{1}^{-}$, each multiplied by an indicator dummy that equals one depending upon whether the plant expands or contracts employment, respectively, and zero otherwise.

\footnotetext{
${ }^{7}$ Imperfect competition can also be allowed for, provided the markup is constant. In that case, marginal profitability is proportional to the sales to labor ratio, with a constant of proportionality that equals the elasticity of output with respect to labor, multiplied by the inverse of one plus the mark-up of prices over marginal costs.

${ }^{8}$ See, for instance, Abel and Blanchard (1986) and Gilchrist and Himmelberg (1999) in the context of investment models with quadratic adjustment costs that are a function of both investment and the capital stock. The idea is that the derivative with respect to the capital stock is a function of the investment rate squared, which is likely to be small if the investment rate is not too big.
} 
Given all these assumptions, we will write the shadow value of employment as:

$$
q_{i t}=\gamma_{0}+\gamma_{1}{ }^{\prime} Z_{i t}-\varepsilon_{i t}
$$

where $Z_{i t}$ includes present and/or lagged values of the sales to labor ratio and of the average wage rate per worker. Note that we have added an error term $\varepsilon_{t i}$ to the definition of the shadow value of employment to capture all those idiosyncratic factors at the plant level that are not observable by the econometrician. We will assume that $\varepsilon_{i t}$ is normally independently distributed with mean zero and variance $\sigma_{\varepsilon}^{2}$. The presence of $\varepsilon_{i t}$ introduces, in the simplest of ways, an error term in the two employment change equations, (3) and (3').

Parameters estimates for the model summarized by (3), (3'), (4), (4') and (6) can be obtained in several ways. ${ }^{9}$ In this paper we will employ a Heckman type two-step estimator. Computational simplicity is one of the attractive features of this procedure. First we estimate the Ordered Probit model for the probability of expanding, maintaining unchanged, or contracting employment to obtain the determinants of the shadow value of employment. Then we estimate the first order conditions for employment expansions and contractions, (3) and (3'), with the appropriate selection correction, using the estimate of the shadow value of employment obtained from the Ordered Probit model. More specifically, the Ordered Probit model can be written as:

\footnotetext{
${ }^{9}$ Note that we are using the information on which regime the plant is in (positive employment changes, negative employment changes, no changes). The model estimated in Hamermesh (1989) and (1992) is instead an unobservable regime model, basically because it is assumed that when plants want to keep employment constant, they succeed only on average (since there is a mean zero error term in the do nothing equation).
} 


$$
\begin{aligned}
& \log L=\sum_{t=1}^{T} \sum_{i \in \Omega_{t}^{+}} \log \Phi\left[\tilde{\gamma}_{1}^{\prime} Z_{i t}+\left(\tilde{\gamma}_{0}-\tilde{b}^{+}\right)-\sqrt{2 \widetilde{c}^{+} \cdot\left(\frac{\tilde{a}_{0}^{+}}{L_{i t-1}}+\tilde{a}_{1}^{+}\right)}\right] \\
& +\sum_{t=1}^{T} \sum_{i \in \Omega_{t}^{-}} \log \left\{1-\Phi\left[\tilde{\gamma}_{1}^{\prime} Z_{i t}+\left(\tilde{\gamma}_{0}+\tilde{b}^{-}\right)+\sqrt{2 \widetilde{c}^{-} \cdot\left(\frac{\tilde{a}_{0}^{-}}{L_{i t-1}}+\tilde{a}_{1}^{-}\right)}\right]\right\} \\
& +\sum_{t=1}^{T} \sum_{i \in \Omega_{t}^{0}} \log \left\{\Phi \left[\left(\widetilde{\gamma}_{1}^{\prime} Z_{i t}+\left(\widetilde{\gamma}_{0}+\widetilde{b}^{-}\right)+\sqrt{2 \widetilde{c}^{-} \cdot\left(\frac{\widetilde{a}_{0}^{-}}{L_{i t-1}}+\tilde{a}_{1}^{-}\right)}\right]\right.\right. \\
& -\Phi\left[\left(\tilde{\gamma}_{1}^{\prime} Z_{i t}+\left(\widetilde{\gamma}_{0}-\widetilde{b}^{+}\right)-\sqrt{2 \widetilde{c}^{+} \cdot\left(\frac{\tilde{a}_{0}^{+}}{L_{i t-1}}+\widetilde{a}_{1}^{+}\right)}\right]\right\}
\end{aligned}
$$

where $\Phi($.$) denotes the standard normal cumulative distribution function. Moreover, \Omega_{t}^{+}, \Omega_{t}^{-}$, $\Omega_{t}^{0}$ denote the set of plants present in the sample at time $t$ that, respectively, expand, contract or maintain unaltered the workforce. Note that a $\sim$ above a parameter denotes the ratio between the original parameter and $\sigma_{\varepsilon}$ (for instance, $\widetilde{c}^{+}=c^{+} / \sigma_{\varepsilon}, \widetilde{c}^{-}=c^{-} / \sigma_{\varepsilon}$, and that the parameters of the cost function can be identified only up to the scale parameter $\sigma_{\varepsilon}$. Furthermore, we are not able to identify the $\gamma_{0}$ from $b^{+}$or $b^{-}$. The Ordered Probit model allows us to recover estimates of $\tilde{\gamma}_{1}, \tilde{\gamma}_{0}-\widetilde{b}^{+}, \widetilde{\gamma}_{0}+\widetilde{b}^{-}, \widetilde{c}^{+} \widetilde{a}_{0}^{+}, \widetilde{c}^{+} \widetilde{a}_{1}^{+}, \widetilde{c}^{-} \widetilde{a}_{0}^{-}, \widetilde{c}^{-} \widetilde{a}_{1}^{-}$. The estimate for $\tilde{\gamma}_{1}$ can be used to construct a proxy for $q_{i t}$ and, together with those of the other parameters, allow us to estimate the expected value of the error terms in the employment change equations, conditional on the probability of being in an employment increase or employment decrease regime.

In the second step, therefore, we estimate the following two equations:

$$
\frac{\Delta L_{i t}}{L_{i t-1}}=\frac{\left(\widetilde{\gamma}_{0}-\widetilde{b}^{+}\right)}{\widetilde{c}^{+}}+\frac{1}{\widetilde{c}^{+}}\left(\widetilde{\gamma}_{1}^{\prime} Z_{i t}+\lambda_{i t}^{+}\right)+\eta_{i t}^{+}
$$


for employment increases and:

$$
\frac{\Delta L_{i t}}{L_{i t-1}}=\frac{\left(\widetilde{\gamma}_{0}+\widetilde{b}^{-}\right)}{\widetilde{c}^{-}}+\frac{1}{\widetilde{c}^{-}}\left(\widetilde{\gamma}_{1}^{\prime} Z_{i t}-\lambda_{i t}^{-}\right)+\eta_{i t}^{-}
$$

for employment decreases. The terms $\eta_{i t}^{+}$and $\eta_{i t}^{-}$denote zero means error terms, while $\lambda_{i t}^{+}$and $\lambda_{i t}^{-}$denote the appropriate inverse Mills ratios and are defined as:

$$
\begin{aligned}
& \lambda_{i t}^{+}=\frac{\phi\left[\tilde{\gamma}_{1}^{\prime} Z_{i t}+\left(\tilde{\gamma}_{0}-\tilde{b}^{+}\right)-\sqrt{2 \widetilde{c}^{+} \cdot\left(\frac{\tilde{a}_{0}^{+}}{L_{i t-1}}+\tilde{a}_{1}^{+}\right)}\right]}{\Phi\left[\tilde{\gamma}_{1}^{\prime} Z_{i t}+\left(\tilde{\gamma}_{0}-\tilde{b}^{+}\right)-\sqrt{2 \widetilde{c}^{+} \cdot\left(\frac{\tilde{a}_{0}^{+}}{L_{i t-1}}+\tilde{a}_{1}^{+}\right)}\right]} \\
& \lambda_{i t}^{-}=\frac{\phi\left[\tilde{\gamma}_{1}^{\prime} Z_{i t}+\left(\tilde{\gamma}_{0}+\tilde{b}^{-}\right)+\sqrt{2 \widetilde{c}^{-} \cdot\left(\frac{\tilde{a}_{0}^{-}}{L_{i t-1}}+\tilde{a}_{1}^{-}\right)}\right]}{1-\Phi\left[\tilde{\gamma}_{1}^{\prime} Z_{i t}+\left(\tilde{\gamma}_{0}+\widetilde{b}^{-}\right)+\sqrt{2 \widetilde{c}^{-} \cdot\left(\frac{\tilde{a}_{0}^{-}}{L_{i t-1}}+\tilde{a}_{1}^{-}\right)}\right]}
\end{aligned}
$$

where $\phi($.$) denotes the standard normal density function. Equations (8) and (8') are estimated$ by OLS after replacing $\tilde{\gamma}_{1}{ }^{\prime}, \lambda_{i t}^{+}$and $\lambda_{i t}^{-}$with the values constructed using the estimates obtained using the in the ordered probit model. Since size, $L_{i t}$, does not enter in the equation for net employment changes, but it enters the thresholds, we have now a useful exclusion restriction that can help us identifying the employment changes equations when we estimate 
them using this Heckman type of procedure. ${ }^{10}$ Under the assumption that the variables in $Z_{i t}$ are uncorrelated with $\varepsilon_{i t}$, the ordered probit yields consistent estimates of the parameters (or functions thereof) listed after equation (7) and of their standard errors. OLS estimation of (8) and $\left(8^{\prime}\right)$ yields consistent estimates of the parameters (if the $Z_{i t}^{\prime s}$ are uncorrelated with the $\eta_{i t}^{\prime s}$ ), but not of their standards errors, because of the well-known generated regressor problem. However, since there is only one generated regressor in each equation, the estimated value of $\left(\tilde{\gamma}_{1}^{\prime} Z_{i t}+\lambda_{i t}^{-}\right)$and $\left(\tilde{\gamma}_{1}^{\prime} Z_{i t}-\lambda_{i t}^{-}\right)$respectively, the $t$-statistics to test the hypothesis that their coefficient is zero is valid (see Pagan (1984)). More importantly, given estimates of $\widetilde{c}^{-}$and $\widetilde{c}^{+}$from the second stage regressions, we can recover point estimates of $\widetilde{a}_{0}^{+}, \widetilde{a}_{1}^{-}, \widetilde{a}_{0}^{+}$and $\widetilde{a}_{1}^{-}$. In order to sharpen our inference, we will calculate the bootstrap value of the confidence intervals for $\tilde{c}^{+}, \widetilde{c}^{-}, \tilde{a}_{0}^{+}, \tilde{a}_{1}^{+}, \widetilde{a}_{0}^{-}, \tilde{a}_{1}^{-} \cdot{ }^{11}$

The model summarized by (3), (3'), (4), (4') and (6) could also be estimated by Maximum Likelihood. The log likelihood function can be written as:

\footnotetext{
${ }^{10}$ See also Letterie and Pfann (2002) for a maximum likelihood application to investment and Lewbel (2002) for a semiparametric estimator of the second stage equation that relies on this exclusion restriction, also with an application to investment.

${ }^{11}$ In estimating the Ordered Probit model we have forced $\widetilde{c}^{+} \widetilde{a}_{0}^{+}, \widetilde{c}^{+} \widetilde{a}_{1}^{+}, \widetilde{c}^{-} \widetilde{a}_{0}^{-}, \widetilde{c}^{-} \widetilde{a}_{1}^{-}$to be positive during the iterations (using an exponential transformation) in order to ensure that the argument of the square-root described in eq. (7) is positive. Note however, that we impose no restriction on $\widetilde{c}^{+}$and $\widetilde{c}^{-}$in estimating equations (8) and ( $\left.8^{\prime}\right)$. This implies that $\widetilde{a}_{0}^{+}, \widetilde{a}_{1}^{-}, \widetilde{a}_{0}^{+}$and $\widetilde{a}_{1}^{-}$could be negative.
} 


$$
\begin{aligned}
& \log L= \sum_{t=1}^{T} \sum_{i \in \Omega_{t}^{+}}\left(\log \widetilde{c}^{+}-0.5 \cdot \log (2 \Pi)-0.5 \cdot\left(\frac{\Delta L_{i t}}{L_{i t-1}} \cdot \widetilde{c}^{+}-\left(\widetilde{\gamma}_{0}-\widetilde{b}^{+}\right)-\widetilde{\gamma}_{1}^{\prime} Z_{i t}\right)^{2}\right) \\
&+ \sum_{t=1}^{T} \sum_{i \in \Omega_{t}^{-}}\left(\log \widetilde{c}^{-}-0.5 \cdot \log (2 \Pi)-0.5 \cdot\left(\frac{\left.\left.\Delta L_{i t} \cdot \widetilde{c}^{-}-\left(\widetilde{\gamma}_{0}+\widetilde{b}^{-}\right)-\widetilde{\gamma}_{1}^{\prime} Z_{i t}\right)^{2}\right)}{+}+\sum_{t=1}^{T} \sum_{i \in \Omega_{t}^{0}} \log \left\{\Phi\left[\widetilde{\gamma}_{1}^{\prime} Z_{i t}+\left(\widetilde{\gamma}_{0}+\widetilde{b}^{-}\right)+\sqrt{2 \widetilde{c}^{-} \cdot\left(\frac{\widetilde{a}_{0}^{-}}{L_{i t-1}}+\widetilde{a}_{1}^{-}\right)}\right]\right.\right.\right. \\
&\left.-\Phi\left[\widetilde{\gamma}_{1}^{\prime} Z_{i t}+\left(\widetilde{\gamma}_{0}-\widetilde{b}^{+}\right)-\sqrt{2 \widetilde{c}^{+} \cdot\left(\frac{\tilde{a}_{0}^{+}}{L_{i t-1}}+\widetilde{a}_{1}^{+}\right)}\right]\right\}
\end{aligned}
$$

However, we have not had much success with the ML procedure. We have encountered severe convergence problem and we were unable to estimate with any precision the fixed adjustment cost parameters. Even when convergence was apparently achieved, the point estimates implied unreasonable values of the estimated probabilities of each regime. ${ }^{12}$

\section{Results}

In this section we will report estimation result for the model summarized by (7), (8), (8'), (9) and (9'). We have estimated various versions of the model based on different assumptions about the information set used by plants in forecasting the present value of returns to one additional worker and with different specifications of the fixed components of adjustment costs. In one specification (see Table 2, Column 1) plants are assumed to have contemporaneous information about sales during period $\mathrm{t}, S_{t}$, and the real wage per worker,

\footnotetext{
${ }^{12}$ For instance, the estimated probability of the inaction regime was very close to one, while the ones for the employment expansion and contraction regimes were very close to zero. One conjecture is that in the full likelihood model, the thresholds parameters are basically pinned down only by the zero observations (see equation (10)), while in the Ordered Probit model (see eq. (7)) the positive and negative employment changes observations contribute to their determination.
} 
$w_{t}$, and $Z_{t}$ is defined as $Z_{t}=\left[\left(S_{t} / L_{t-1}\right),\left(S_{t-1} / L_{t-2}\right), w_{t}, w_{t-1}\right]$, where we are omitting the $i$ subscript for simplicity throughout this section. We have experimented with dividing $S_{t}$ by $L_{t}$ or by $L_{t-1}$, and we report the results using the latter specification. ${ }^{13}$ In another specification, (see Table 2, Column 2) we do not include any contemporaneous variable and $Z_{t}$ is defined as $Z_{t}=\left[\left(S_{t-1} / L_{t-2}\right),\left(S_{t-2} / L_{t-3}\right), w_{t-1}, w_{t-2}\right]$. Results for the general specification of adjustment costs contained in equation (2) are reported in Table 2. In both the ordered probit model and employment equations we include three digit sectoral dummies, year dummies, plant type dummies, a size dummy ( $>50$ employees), and a dummy for the year of creation of the plant. ${ }^{14}$

The Ordered Probit results in Column 1 suggest that our proxy for the shadow value of employment depends significantly both upon the sales to labor ratio and upon the wage rate. More specifically, the coefficient of $S_{t} / L_{t-1}$ is positive significant and much larger (in absolute value) than the negative coefficient of $S_{t-1} / L_{t-2}$. The coefficient of the contemporaneous wage is positive but insignificant, while the lagged wage has negative coefficient that is larger in absolute value and significant. This means that, looking at the sum of the coefficients on the contemporaneous and lagged determinants of $q_{t}$, the sales to labor ratio has a positive effect and the wage a negative effect on the shadow value of labor $q_{t}$, as one would expect. As a result, a (permanent) higher sales to labor ratio is associated with an increase in the probability of observing an increase in employment, while a (permanently)

\footnotetext{
${ }^{13}$ Strictly speaking, in the model of Section 4, production depends upon $L_{t}$, so that division by $L_{t}$ may appear more appropriate. Division by $L_{t-1}$ is would be more appropriate if workers become productive with one period delay. In this case, the model would be very similar, but the precise definition of the shadow value of employment to which the quadratic components of adjustment costs is equated, when expanding or contracting, would be slightly different. More precisely: $q_{t}=E_{t} \sum_{j=0}^{\infty} \beta^{j+1}\left[F^{\prime}\left(A_{t+j}, L_{t+j}\right)-\beta^{-1} w_{t+j}-\beta \cdot G_{L}\left(\Delta L_{t+j+1}, L_{t+j}\right)\right]$. In the data, employment is measured in the middle of the year.

${ }^{14}$ Note that the inclusion of the size dummy does not affect our conclusions regarding the parameters of the adjustment cost function. Its coefficient in the Ordered Probit Model is actually not significant and the results do not change if its coefficient is set to zero.
} 
higher wage is associated with a decrease in such probability. The opposite holds true for the probability of employment decreases. The average (across plants and time) estimated probability of the employment expansion, employment contraction, and no change regimes equal $0.384,0.408$ and 0.208 respectively. These figures make sense, given the descriptive statistics reported in Table 1.

Estimation of the employment change equation with the selection correction, suggests that our proxy for the shadow value of an additional worker is significantly related to both employment increases and decreases. The point estimate for $\widetilde{c}^{+}$is 5.238 and for $\widetilde{c}^{-}$is 5.244 . In order to assess the significance of the quadratic components of adjustment costs we also report the bootstrap $95 \%$ confidence intervals for $\widetilde{c}^{+}$and $\widetilde{c}^{-}$. In both cases the estimates are well bounded away from zero. ${ }^{15}$ Note that in this specification there is no evidence of asymmetries in the shape of the convex elements of adjustment costs.

The estimates of the fixed costs parameters imply that it is the pure fixed costs components, that are the more precisely determined, while those proportional to the existing stock of workers are less precisely determined. While the estimates of $\tilde{a}_{0}^{+}$and $\widetilde{a}_{0}^{-}$are well bounded away from zero, those for $\tilde{a}_{1}^{+}$and $\widetilde{a}_{1}^{-}$are not (the $2.5^{\text {th }}$ percentile is actually a miniscule positive number in both cases). Similarly, the coefficients of $\widetilde{c}^{+} \widetilde{a}_{0}^{+}$and $\widetilde{c}^{-} \widetilde{a}_{0}^{-}$are significantly or nearly so in the probit model, while those for $\widetilde{c}^{+} \widetilde{a}_{1}^{+}$and $\widetilde{c}^{-} \widetilde{a}_{1}^{-}$are completely insignificant. In terms of point estimates, it appears that it is particularly costly to fire workers, since $\tilde{a}_{0}^{-}$is one sixth the size of $\widetilde{a}_{0}^{+}$.

All this suggests that plant size matters in determining the threshold values of $q_{t}$ beyond which the plant decides to increase or decrease employment. The range over which

\footnotetext{
${ }^{15}$ The bootstrap confidence intervals are based on 1000 replications for the Ordered Probit model and for the of the employment equations.
} 
plants keep employment constant is wider for plants with smaller initial employment, because both the lower threshold decreases and the upper threshold increases. The effect of size is much larger on the lower threshold. These overall econometric results seem to be very much consistent with the descriptive evidence discussed in Section 3, and in particular, with the larger frequency of zero employment changes episodes for smaller plants.

The second column of Table 2 contains the results obtained when only lagged information is used in forming a proxy for the shadow value of employment. This reduces the potential endogeneity problems due to the correlation between contemporaneous sales and possibly wages and the error term. Most of the previous conclusions still hold. Sales continue to play the important role implied by the results of Column 1. However, now the coefficients of wages lagged once or twice are not significant, and while opposite in sign, they are very close in absolute value. The purely fixed components of adjustment costs, $\widetilde{a}_{0}^{+}$and $\widetilde{a}_{0}^{-}$, appear again to be important, particularly the one associated with employment reductions, $\widetilde{a}_{0}^{-}$. One difference is that the point estimate of $\widetilde{c}^{-}$exceeds the one for $\widetilde{c}^{+}(8.089$ versus 5.270$)$. This suggests that it is more costly to adjust employment at the margin in a downward direction.

In Column 3 we have attempted to control for the presence of plants' fixed effects in the ordered probit and hiring and firing equations. More specifically, we have included in the model of Column 2 the average value of the sales to capital ratio and of the wage rate, both in the Ordered Probit model and in each employment growth equations. This is tantamount to assuming that the plant fixed effect can be modeled as a linear combination of these two variables. In order to minimize endogeneity problems, we have taken the average over the first three years of observation and used the remaining years for estimation. ${ }^{16}$ Again sales play an

\footnotetext{
${ }^{16}$ We use the same sample for estimation in all the specifications of Table 2. For the first two columns that do not contain the average wage rate and sales to capital ratio, one could use the initial years for estimation. When this is done the results are very similar and are not reported here.
} 
important role in the determination of the shadow value of an additional worker. The effect of the wage is not precisely estimated, although now the negative coefficient $w_{t-1}$ exceeds in absolute value the positive coefficient of $w_{t-2}$. The purely fixed components of adjustment costs, and not those proportional to the stock of workers, are still the ones that have a significant effect, particularly $\widetilde{a}_{0}^{-}$which remains also much larger than $\widetilde{a}_{0}^{+}$. Finally, also in this case the estimate of $\widetilde{c}^{-}$exceeds the one for $\widetilde{c}^{+}$, suggesting an asymmetry of the quadratic component. On the basis of the $95 \%$ bootstrap confidence interval (or of the standard error) of $\tilde{a}_{0}^{-}-\tilde{a}_{0}^{+}$, we can reject the equality of the fixed components during employment contractions and expansions. However, the same statistics for $\widetilde{c}^{-}-\widetilde{c}^{+}$do not allow us to reject the equality of the quadratic parameters in the two regimes. ${ }^{17}$

We have already commented on the fact that $\tilde{a}_{1}^{+}$and $\widetilde{a}_{1}^{-}$are not precisely estimated. In Column 4 we report the results obtained when $\tilde{a}_{1}^{+}$and $\widetilde{a}_{1}^{-}$are set equal to zero. ${ }^{18}$ The likelihood ratio test of this restriction equals 4.42 , with a marginal significance level close to $10 \%$. As in Column 3 we include the average values of the wage rate and of the sales to capital ratio. The qualitative conclusions we have reached on the basis of the specification in Column 3 remain mostly the same. The purely fixed component when reducing employment is significant and larger than the one for expanding employment. However, now it is smaller in magnitude compared to its value in Column 3. The estimates of the quadratic components remain very similar to those in Column 3

\footnotetext{
${ }^{17}$ The same is true for the other specifications in Table 2.

${ }^{18}$ If $\widetilde{a}_{1}^{+}$and $\widetilde{a}_{1}^{-}$are zero, the specification of the shadow value of employment we have used is a better approximation to its true value, because it only omits the derivative of the quadratic component of adjustment costs with respect to the stock of workers.
} 
Since the adjustment cost parameters are estimated relative to the variance of the error term, it is not possible to make unambiguous statements about the absolute size of adjustment costs. However, we can assess the quantitative importance of the different components of adjustment costs relative to one another. This is a very interesting issue that our model can help shedding light on, although the results vary somewhat across specifications. In Figure 1a we have plotted, for different plant sizes, the value of the employment expansion or contraction rate that makes the size of the quadratic and fixed components equal to each other, on the basis of the estimates of Column 3. For instance, for a plant of 100 employees the quadratic components in a contraction equals the sum of the fixed components (the purely fixed one plus the one proportional to past employment) when the employment decreases at a rate of approximately $30 \%$ per year. For employment contractions greater than $30 \%$ the quadratic component is greater than the fixed components. When expanding, the fixed and quadratic components are of equal size for an employment growth rate of approximately $40 \%$. Both figures increase as the plant size decreases, reflecting the presence of a purely fixed component. These results suggest a dominant fixed component, relative to the quadratic component, for most rates of change (positive or negative) experienced by plants. However, these conclusions are misleading because it is driven by the point estimates of the fixed component proportional to size, which is very imprecisely determined.

In Figure $1 \mathrm{~b}$ we still use the estimates from Column 3, but look for the values of $\Delta L_{t} / L_{t-1}$ that equate the quadratic component to the purely fixed component. In this case the employment rates need to be around 10\% for employment contractions and 5\% for expansions.

Finally, in Figure 1c we report the results for the model of Column 4, in which $\widetilde{a}_{1}^{+}$and $\widetilde{a}_{1}^{-}$are set equal to zero. In this case fixed costs and quadratic adjustment costs are of equal size for plants with 20 workers at employment contraction rates of approximately $10 \%$ or at employment expansion rates of around $2.5 \%$. For plants of 100 workers or more, the figures 
are $5 \%$ for contractions and very small for expansions. The bottom line is that for a small plant the quadratic components are always important. The purely fixed component is also important when employment contracts. For large plants the quadratic components are the dominant ones. In both cases, contracting is more costly than expanding at the margin.

A final issue worth addressing is how well the model does in predicting the aggregate investment rate for the plants in metal products and machinery industry. In Figure $2 \mathrm{a}$ we plot the aggregate net job creation (destruction rate) calculated summing over the sample of plants we have used in estimating the model (denoted by NJCR and reported on the right hand axis). We also plot the average ex-ante probability of an employment expansion, of an employment contraction, and the probability of no employment change, calculated using the estimated parameters of our model (see left hand axis). ${ }^{19}$ We observe that the average expansion probability tracks quite well the proportional change of aggregate employment, capturing all the turning points. The average probability of employment decreases moves, instead, strongly counter-cyclically relative to aggregate employment changes. The average probability of no employment change fluctuates less, but displays a counter-cyclical pattern as well. As a result recessions appear to be periods in which the probability of employment expansions decrease on average, while the probability of employment contractions and of no employment change both increase, although the movements in the former are more pronounced.

In Figure $2 \mathrm{~b}$, we use our model to calculate the predicted proportional employment change based on the parameter estimates for our model. Calculation of the predicted employment change for each plant at each point in time is based on the estimates of employment equations in the second stage, but also takes into account that the expected value

\footnotetext{
${ }^{19}$ We have calculated the probability of each regime for each individual plant at a given point in time, using the parameter estimates of the Ordered Probit model. We have then taken the unweighted average of these probabilities across plants, for each $t$.
} 
of the error term is not zero. The predicted proportional employment change for each plant is then aggregated across plants for each $t$, using lagged plant employment relative to total lagged employment as weights. The resulting predicted net job creation rate is reported in Figure $2 \mathrm{~b}$ together with the actual value. Again we observe that the two series move pretty much together. Aggregate employment expansion in 1991 is over-predicted by the model, but the subsequent fall and recovery after 1993 are quite well captured.

\section{Conclusions}

The results we have presented suggest that the $q$ model of employment with a general specification of adjustment costs seems to be a useful way to organize the analysis of employment changes at the plant level. The initial results imply that it is important to depart from the standard specification of convex and symmetric adjustment costs. Fixed costs are important factors that the plant must consider when changing employment. The evidence suggests the existence of a purely fixed component, unrelated to plant size. As a result, the area of inaction is greater for smaller plants, which is consistent with the greater frequency of inaction episodes observed for them. Moreover, it appears that fixed costs are significantly larger when plants reduce their workforce. The fixed components proportional to size are not, instead, precisely estimated. Quadratic components of costs are also important. The point estimates in most specifications suggest that marginal adjustment costs are higher during employment contractions compared to expansions, although the difference is not significant. The fact that fixed and quadratic components of adjustment costs appear to be important when plants reduce their workforce is consistent with our understanding of the job protection legislation and practices in Norway. 
There are a set of issues we have not addressed and that need to be explored in future work. In particular, it would be useful to analyze the interaction between labor and capital adjustment costs. It would also be interesting to analyze how adjustment costs differ across different categories of workers, according to their skill level, for instance. Finally the issue of whether adjustment costs are related to net or gross employment changes, or both, deserves further attention. Still, the evidence provided in this paper shows the importance and potential fruitfulness of departing from traditional models of labor demand based solely on symmetric convex adjustment costs. 


\section{References}

Abel, A. and O. Blanchard, 1986, "The Present Value of Profits and Cyclical Movements in Investment”, Econometrica 54(2), 249-273.

Abel, A. B. and J.C. Eberly, 1994, "A Unified model of Investment Under Uncertainty", American Economic Review, 84, 1369-1384.

Abel, A. B. and J.C. Eberly, 1999, "Investment and q with Fixed Costs: an Empirical Analysis", mimeo, The Wharton School, University of Pennsylvania, April.

Abowd, J. M., and F. Kramarz, 2003, "The Cost of Hiring and Separations", Labour Economics 10, 499-530.

Abowd, J. M., P. Corbel and F. Kramarz, 1999, "The entry and exit of workers and the growth of employment: An analysis of French establishments", The Review of Economics and Statistics 81(2), 170-187.

Barnett, S. A. and P. Sakellaris, 1998, "Non-Linear Response of Firm Investment to Q: Testing a Model of Convex and Non-Convex Adjustment", Journal of Monetary Economics 42(2), 261-288.

Bentolila, S. and G. Saint-Paul, 1994, "A Model of Labor Demand with Linear Adjustment Costs", Labour Economics 1, 303-326.

Campbell J.R. and J.D.M. Fisher (2000a), "Aggregate Employment Fluctuations with Microeconomic Asymmetries", American Economic Review 90, 1323-1345.

Campbell J.R. and J.D.M. Fisher (2000b), "Idiosyncratic Risk and Aggregate Employmnet Dynamics", National Bureau of Economic Research, Working Paper No. 7936

Cooper, R., J. Haltiwanger and L. Power, 1999, "Machine Replacement and the Business Cycle: Lumps and Bumps", American Economic Review 89(4), 921-946.

Doms, M., and T. Dunne, 1998, "Capital Adjustment Patterns in Manufacturing Plants", Review of Economic Dynamics 1, 409-429.

Emerson, M. (1987), "Labour Market Flexibility and Jobs: A Survey of Evidence from OECD Countries with Special Reference to Europe: Comments", in Layard, R. and L. Calmfors (ed.) The fight against unemployment: Macroeconomic papers from the Centre for European Policy Studies. MIT Press, 77-84.

Gilchrist, S. and C. Himmelberg, 1999, "Investment: Fundamentals and Finance", in NBER macroeconomics annual1998 (eds. B. Bernanke, and J. Rotemberg), Cambridge and London: MIT Press, 223-262. 
Goolsbee, A. and D. B. Gross, 1997, "Estimating Adjustment Costs with Data on Heterogeneous Capital Goods", National Bureau of Economic Research, Working Paper No. 6342.

Goux, D., E. Maurin and M. Pauchet, 2001, "Fixed-term contracts and the dynamics of labour demand", European Economic Review 45(3), 533-552.

Halvorsen, R., R. Jenssen and F. Foyn, 1991, "Dokumentasjon av industristatistikkens tidsseriebase", Oslo: Statisitics Norway.

Hamermesh, D. S., 1989, "Labor Demand and the Structure of Adjustment Costs", American Economic Review 79(4), 674-689.

Hamermesh, D. S., 1992, “A General Model of Dynamic Labor Demand”, The Review of Economics and Statistics 74(4), 733-737.

Hamermesh, D. S., 1995, "Labor Demand and the Source of Adjustment Costs", The Economic Journal 105, May, 620-634.

Hamermesh, D.S. and G. Pfann, 1996, "Adjustment costs in factor demand", Journal of Economic Literature 34, 1264-1292.

Kramarz, F. and M.-L. Michaud, 2002, “The Shape of Hiring and Separation Costs”, mimeo, CREST-INSEE.

Letterie, W., and G. Pfann, 2002, "On the Identification of Nonlinearities in Equipment Investment”, mimeo, BIRC, Maastricht University.

Lewbel, A., 2002, “Endogenous Selection and Treatment Model Estimation”, Boston College, Department of Economics, Working Paper No. 462.

OECD, 1999, Employment Outlook, OECD, Paris.

Nilsen, Ø. A. and F. Schiantarelli, 2003, “Zeros and Lumps in Investment: Empirical Evidence on irreversibilities and Non-convexities", forthcoming Review of Economics and Statistics 85(4).

Pagan, A., (1984). "Econometric Issues in the Analysis of Regressions with Generated Regressors, International Economic Review 25, 221-247.

Rota, P., 1995, "Dynamic Labour Demand with Lumpy and Kinked Adjustment Costs", mimeo, University College London.

Salvanes, K.G., 1997, "Market Rigidities and Labour Market Flexibility: An International Comparison", Scandinavian Journal of Economics 99, 315-333. 
Table 1. Descriptive statistics for net employment changes, $\frac{\Delta L_{t}}{L_{t-1}}$

\begin{tabular}{|c|c|c|c|c|c|c|c|}
\hline \multirow{2}{*}{$\frac{\Delta L_{t}}{L_{t-1}}$} & \multirow[b]{2}{*}{ Freq. 1} & \multirow[b]{2}{*}{ Freq. 2} & \multirow[b]{2}{*}{ Share } & \multicolumn{4}{|c|}{ Freq. 1 by plant size } \\
\hline & & & & 4-25 & $26-50$ & $51-100$ & 101- \\
\hline$<-0.5$ & 0.004 & 0.009 & 0.042 & 0.25 & 0.49 & 0.66 & 0.59 \\
\hline$-0.5,-0.4$ & 0.008 & 0.021 & 0.123 & 0.74 & 0.74 & 0.76 & 1.64 \\
\hline$-0.4,-0.3$ & 0.019 & 0.048 & 0.087 & 2.21 & 1.79 & 1.23 & 1.29 \\
\hline$-0.3,-0.2$ & 0.054 & 0.135 & 0.185 & 6.08 & 4.62 & 5.20 & 3.52 \\
\hline $0.2,-0.1$ & 0.133 & 0.332 & 0.319 & 13.69 & 13.87 & 11.15 & 12.79 \\
\hline$-0.1,0.0$ & 0.183 & 0.455 & 0.244 & 10.96 & 24.11 & 31.57 & 32.63 \\
\hline$=0$ & 0.175 & & & 25.22 & 9.93 & 5.39 & 2.35 \\
\hline $0.0,0.1$ & 0.162 & 0.382 & 0.218 & 9.32 & 23.24 & 27.50 & 28.05 \\
\hline $0.1,0.2$ & 0.138 & 0.326 & 0.306 & 15.30 & 13.19 & 10.11 & 11.27 \\
\hline $0.2,0.3$ & 0.057 & 0.134 & 0.145 & 7.46 & 3.51 & 2.93 & 2.93 \\
\hline $0.3,0.4$ & 0.026 & 0.062 & 0.092 & 3.18 & 2.16 & 1.98 & 1.29 \\
\hline $0.4,0.5$ & 0.020 & 0.048 & 0.080 & 2.97 & 1.05 & 0.38 & 0.59 \\
\hline $0.5,1.0$ & 0.018 & 0.042 & 0.124 & 2.42 & 1.05 & 0.76 & 0.94 \\
\hline$>1.0$ & 0.002 & 0.006 & 0.035 & 0.20 & 0.25 & 0.38 & 0.12 \\
\hline Total & $\begin{array}{r}\text { \#obs }= \\
8414\end{array}$ & & & $\begin{array}{r}\text { \#obs }= \\
4881\end{array}$ & $\begin{array}{r}\text { \#obs }= \\
1622\end{array}$ & $\begin{array}{r}\text { \#obs }= \\
1058\end{array}$ & $\begin{array}{r}\text { \#obs }= \\
852\end{array}$ \\
\hline
\end{tabular}

Notes: $\quad$ Freq. 1 denotes the frequency relative to the entire set of observations. Freq. 2 denotes the frequency of each cell of positive (negative) employment changes relative to the subset of positive (negative) employment changes. Share denotes the share of total positive (negative) employment changes accounted for by each cell. 
Table 2. Estimation results

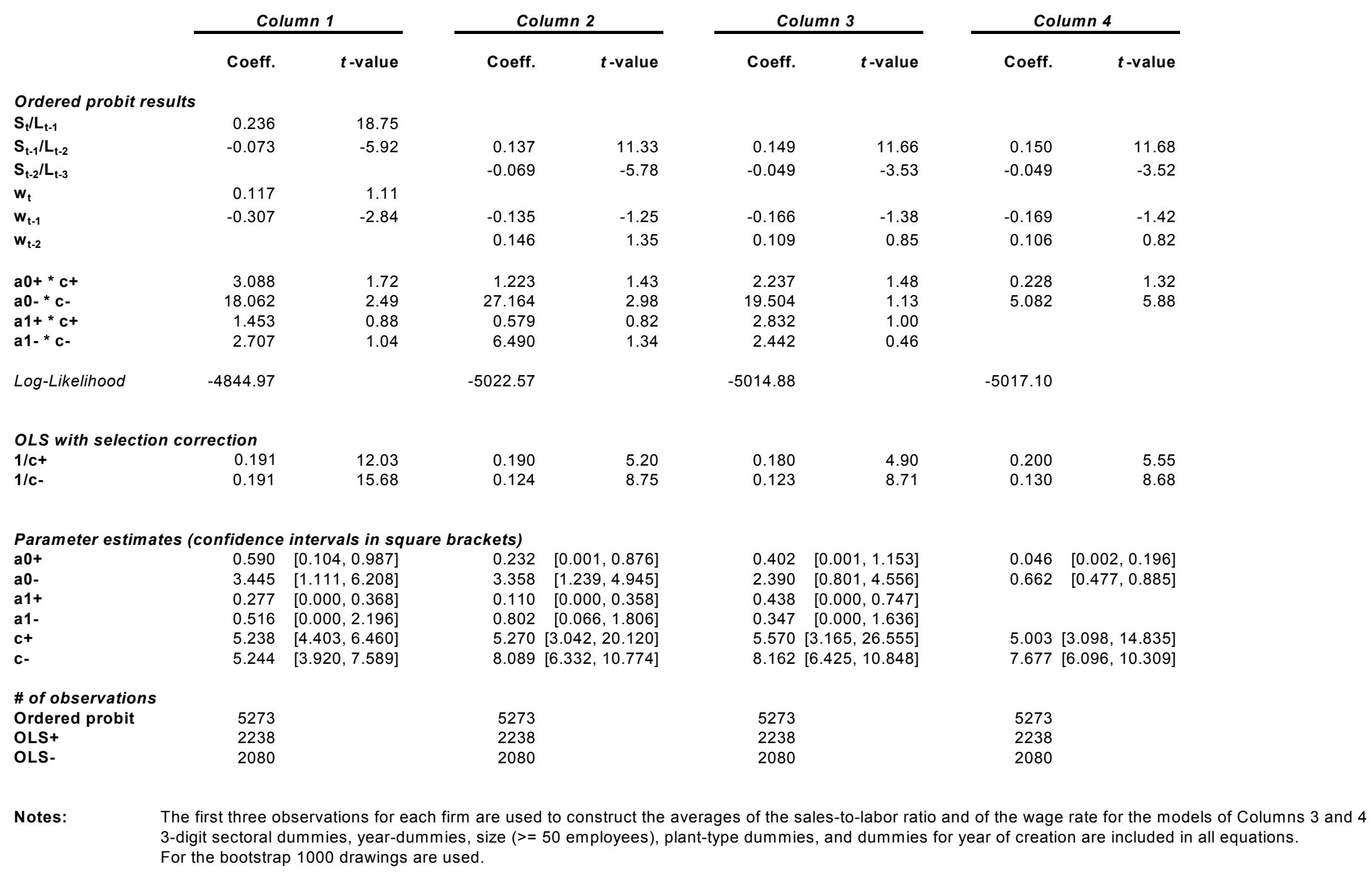


Figure 1: Relative size of fixed and convex costs.

Relative size of total fixed and convex costs

(table 2, col. 3)

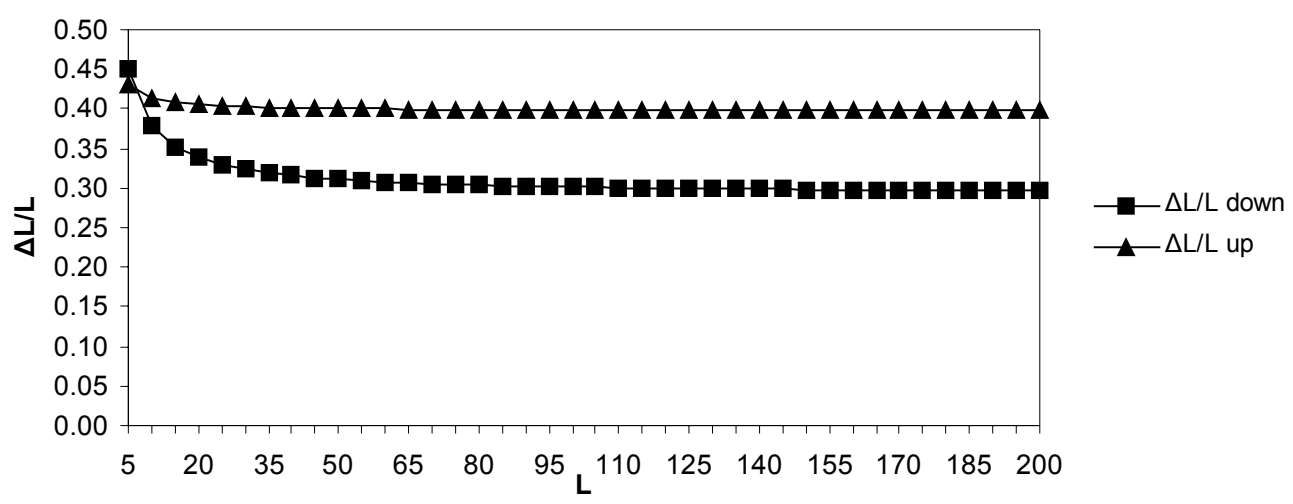

Relative size of purely fixed and convex costs

(table 2, col. 3, a1- = 0, a1+ = 0)

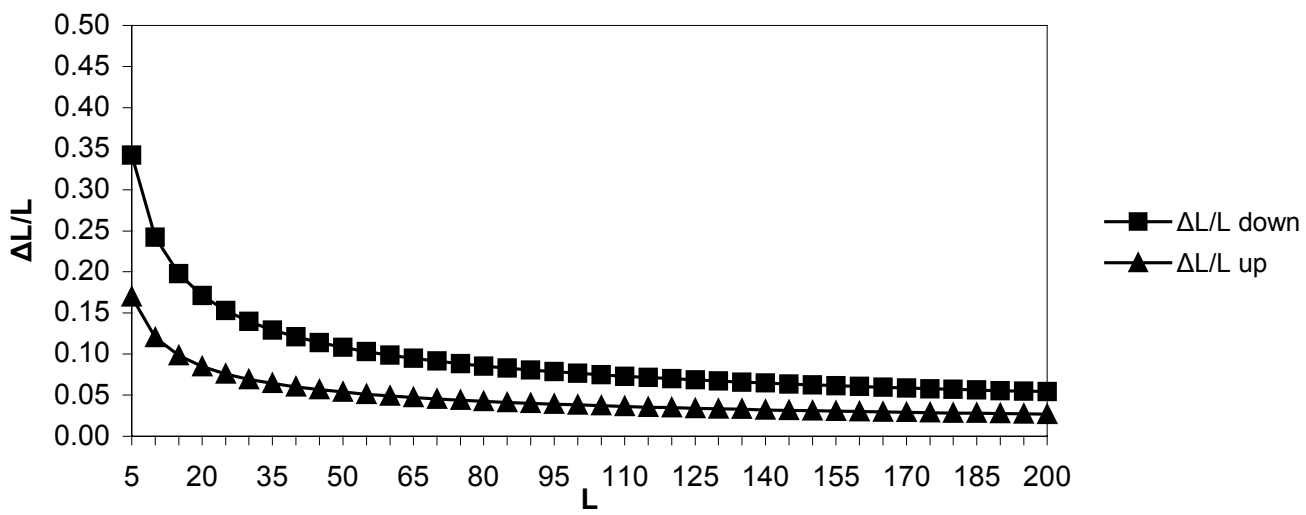

Relative size of total fixed and convex costs (table 2, col. 4, in which a1- = 0 , and $\mathrm{a} 1+=0$ )

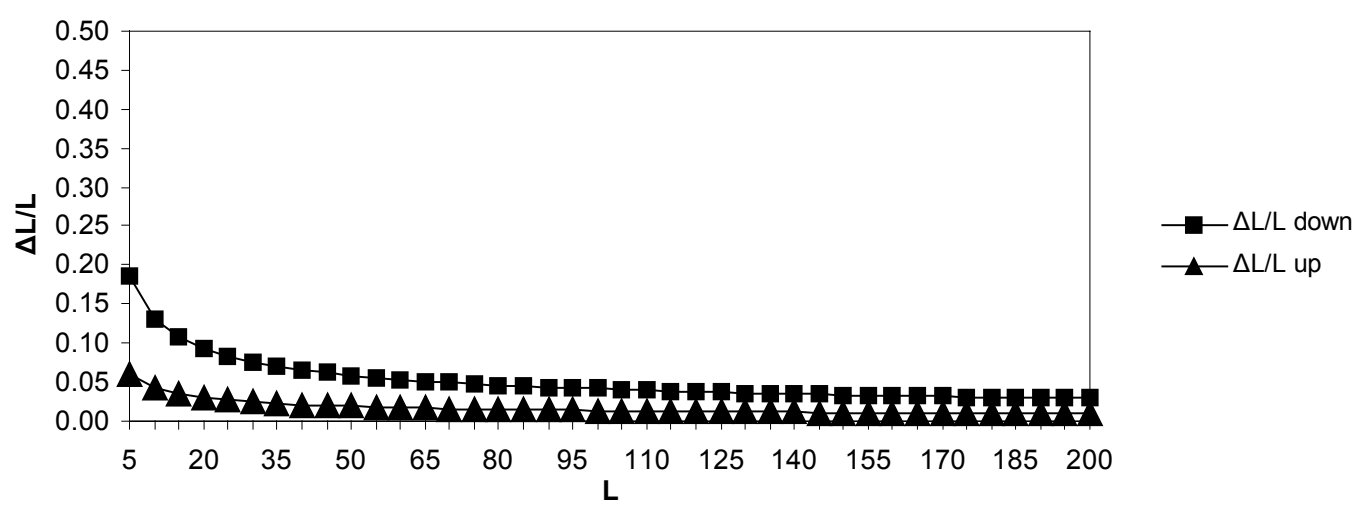

Notes: The lines represent combinations of size and employment growth rates (positive or negative) for which fixed costs and convex costs are equal. 
Figure 2a: Probabilities of employment going up, going down, and remaining the same

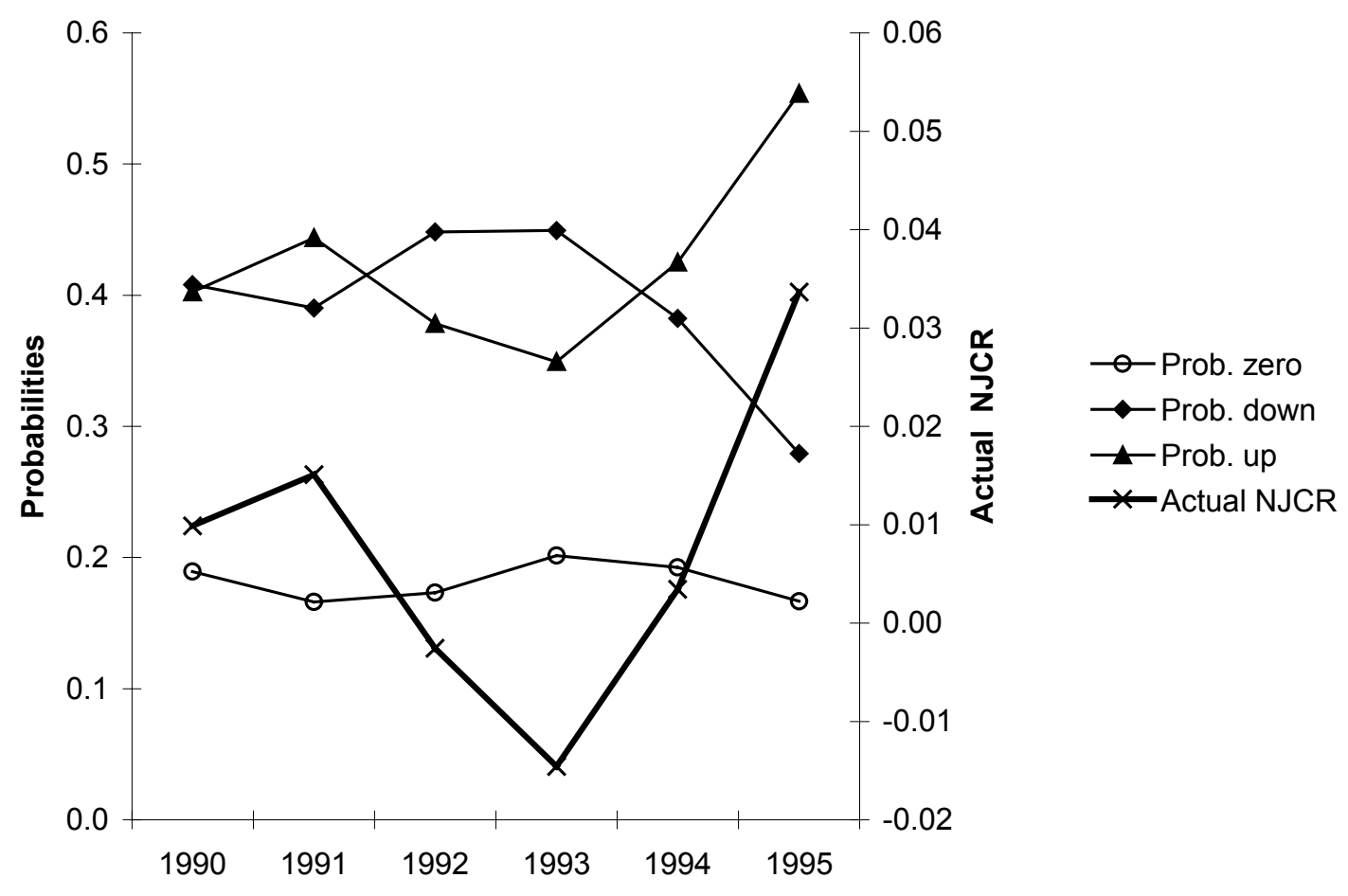

Figure 2b: Actual and predicted net job creation rate (NJCR)

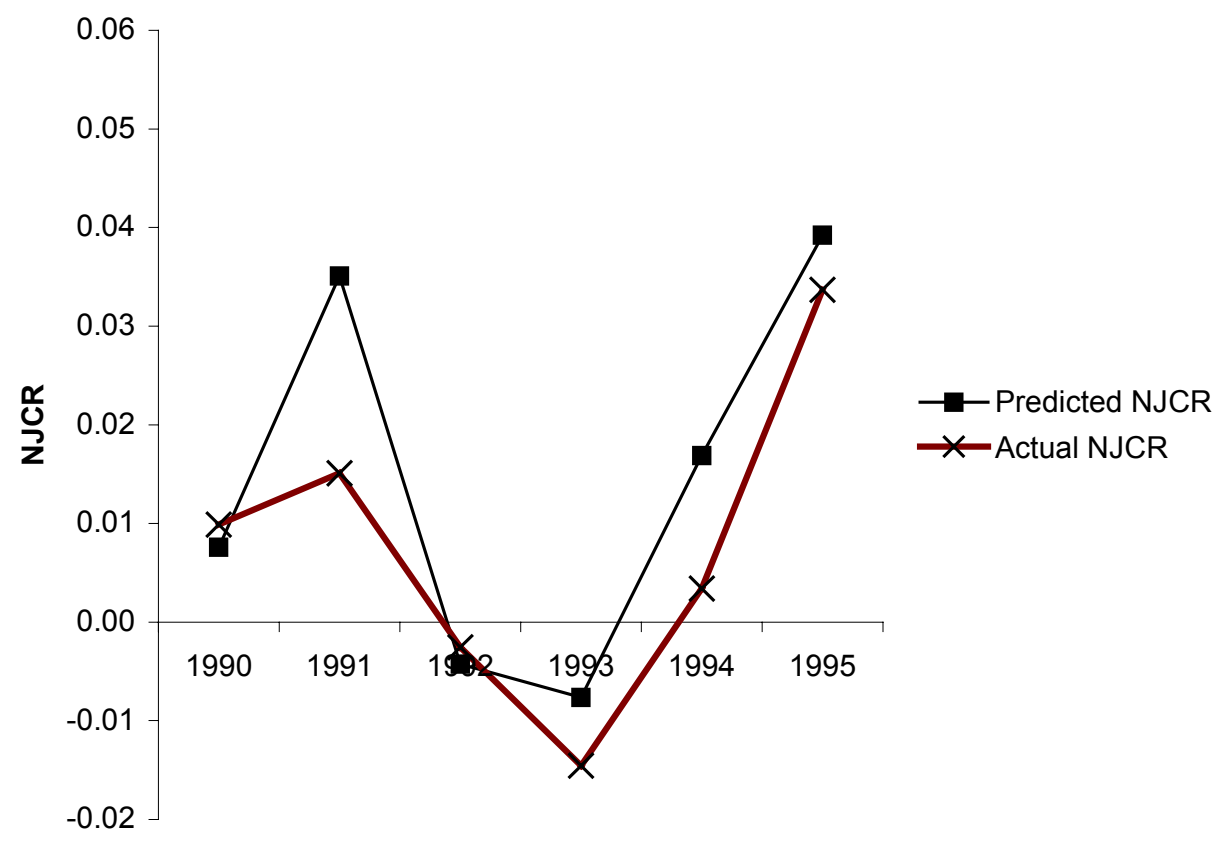

